\title{
Hospital-based HIV/HSV-2 seroprevalence among male patients with anal disease in Korea: cross sectional study
}

\author{
Jin-Sook Wang ${ }^{1,2+}$, Do Yeon Hwang ${ }^{3 \dagger}$, Hye-Kyung Yu', Sung Soon Kim', Jong Kyun Lee ${ }^{3}$ and Mee-Kyung Kee ${ }^{1 *}$
}

\begin{abstract}
Background: This study aimed to identify the characteristics of HIV and herpes simplex virus (HSV)-2 seroprevalence in male patients with anal disease.

Methods: HIV seroprevalence was estimated for different age groups of male patients with anal disease who were treated at Songdo colorectal hospital in Korea between 2001 and 2011. HIV seroprevalence of patients with anal disease was compared with that of patients with nonanal disease for each year from 2007 to 2011 . HSV-2 antibody tests were conducted on 2,038 HIV-tested male patients with anal disease in 2009.

Results: For 11 years from 2001, HIV seroprevalence differed significantly by age group $(P<0.001)$ and was highest in the group aged <20 years. From 2007 to 2011, HIV seroprevalence in patients with anal disease was 7.6/10,000-13.3/ 10,000 and that in patients with nonanal disease was $0-0.9 / 10,000$. HSV-2 seroprevalence among patients with anal disease was 24.0\%, and only one patient with HIV and HSV-2 was observed.

Conclusions: HIV seroprevalencein male patients with anal disease was significantly higher than that for other diseases. HSV-2 seroprevalence was similar to that in the general male population. Implementation of the current HIV surveillance system for male patients at colorectal hospitals is necessary to help prevent HIV transmission.
\end{abstract}

Keywords: HIV/HSV-2, HIV seroprevalence, HSV-2 seroprevalence, Anal disease, Age group

\section{Background}

When AIDS was first reported in 1981, it seemed to be a new disease that affected only homosexual men. However, it was discovered that the disease spread to the general population through drug users, hemophilia patients, children infected by their mothers, and sexual partners of individuals with HIV through blood or sexual contact [1]. Since 1985, when the first case of HIV infection was identified in Korea, the number of HIV infections has continued to increase, with the cumulative total reaching 9,406 in 2012. The ratio of males to females among reported HIV cases is approximately 11:1. Overall, 99\% of HIV infections is caused by sexual contact, and

\footnotetext{
* Correspondence: keemeekyung@gmail.com

${ }^{\dagger}$ Equal contributors

'Division of AIDS, Center for Immunology and Pathology, Korea Centers for Diseases Control and Prevention, Osong Health Technology Adminstration Complex, 187 Osongsaengmyeong2(i)-ro, Cheongwon-gun,

Chungcheongbukdo 363-951, Korea

Full list of author information is available at the end of the article
}

the proportion of cases attributed to male-to-male sexual contact is about $40 \%$ [2]. Almost $70 \%$ of all newly identified cases is identified during hospital care [3]; thus, it was of interest to identify the type of medical procedures and treatment during which the highest number of new HIV diagnoses was made. According to Miles et al. and Wexner et al., 5.9-34\% of the HIV-infected population has anal disorders, which are also the most frequent cause for surgery in this group [4,5]. Among men who have sex with men attending a sexually transmitted infection (STI) clinic, 30.8\% had dysuria, pus, and urethral ulceration, and $37.1 \%$ had pus, bleeding, ulceration, and/or pain in the rectum [6]. A study of HIV infection and anal diseases in Korea found that most HIV infections detected at colorectal hospitals were in men, most of whom were homosexual [7]. Currently, there are more males than females HIV patients and the predominant route of transmission is homosexual contact in Korea.

\section{Biomed Central}


Genital ulcers have been associated with increased risk factors for HIV infection, and genital herpes is usually caused by herpes simplex virus (HSV)-2 [8]. Our previous study had compared HSV-2 seroprevalence in HIVinfected males with that in the general male population in Korea, which was two or three times higher among HIV-infected males [9]. HSV-2 seroprevalence had a tendency to increase with age and HIV seroprevalence had a different characteristic by age in most countries including Korea [9-11].

Given these epidemiological characteristics of HIV infection in Korea, we aimed to estimate HIV seroprevalence and trends among men suffering from anal disease. We also aimed to identify the seroprevalence of these sexually transmitted viruses by measure of HSV-2 infection, a virus that is spread sexually.

\section{Methods}

\section{Subjects}

HIV seroprevalence in male patients who were treated for anal disease at Songdo (S) colorectal hospital from 2001 to 2011 was estimated for different age groups. S colorectal hospital is a representative large hospital specializing in the field of anal diseases in Seoul, Korea. HIV seroprevalence in male patients with anal disease was compared with that in those who presented for other reasons (including a health checkup) calculated for each year from 2007 to 2011. To understand HIV and/or HSV2 seroprevalence, HSV-2 tests were conducted on 2,038 men who received treatment for anal diseases at $\mathrm{S}$ colorectal hospital. The HSV-2 samples randomly selected among 26,173 male patients with anal disease tested for HIV in 2009. Ethics approval was obtained from KCDC Institutional Review Board (IRB) Ethics Committee.

\section{HIV and HSV-2 antibody tests}

HIV screening was performed using Architect HIV AG/AB Combo Kit (Abbott Laboratories, Abbot Park, IL, USA) to determine the presence of HIV antibody and/or antigen. Patient samples that tested positive in HIV screening were subjected to an additional HIV western blot test (HIV BLOT 2.2 Western Blot Assay, MP Diagnostics, Asia Pacific Pte Ltd., Singapore) at an HIV confirmatory testing institute to confirm HIV infection. HSV-2 antibody tests were performed with the HerpeSelect 2 ELISA IgG Kit (Focus Diagnostics, Cypress, CA, USA). HSV-2 results were reported as an index value relative to the cut-off calibrator. An index value $>1.10$ was presumptive for the presence of IgG antibodies to HSV-2.

\section{Statistical analysis}

HIV seroprevalence was defined as the number of confirmed HIV cases per 10,000 HIV-tested people over a 1 -year period. HSV-2 seroprevalence was defined as the proportion of HSV-2-positive people among the total number of people tested. Patients with any type of anal disease were grouped together as the anal disease patients, and patients who visited the hospital for a health checkup or for another intestinal disorder were categorized as the nonanal disease patients. Trend tests were conducted to determine how HIV seroprevalence varied from year to year in the anal and nonanal disease patients, using SAS version 9.3. $X^{2}$ tests were used to compare HIV seroprevalence in patients with anal disease and nonanal disease, and HIV and HSV-2 seroprevalence in different age groups of patients with anal disease, with a $95 \%$ confidence level.

\section{Results}

Status of HIV tests and trends in HIV seroprevalence

S colorectal hospital administered HIV tests to 17,59330,225 male patients with anal disease every year from 2001 to 2011. Approximately 50\% of total HIV tests were performed in patients aged $\geq 50$ years. Overall, 255 HIV-infected males were founded during the same period (data not shown). As shown in Figure 1, annual HIV seroprevalence among male patients with anal disease during the study period was $6.3-16.2$ per 10,000 . The trend revealed that HIV seroprevalence was highest in the group aged $<20$ years, followed by 20-29, 30-39, 40-49 and $\geq 50$ years $(P<0.0001)$. Over 11 years, 1,254 teenagers (approximately $0.5 \%$ of the total) were treated for anal disorders and among these, 10 were diagnosed with HIV (data not shown). The trend in HIV seroprevalence over time did not show any specific pattern in any of the age groups: under 20 ( $P=0.5982), 20-29(P=0.3675), 30-39$ $(P=0.7476), \quad 40-49 \quad(P=0.1336), \quad 50-59 \quad(P=0.0815)$, and $\geq 60(P=0.3709)$ years.

As shown in Table 1, for the trend in HIV seroprevalence among male patients with anal or nonanal disease, 119 were found to be positive for HIV from 2007 to 2011. One hundred and sixteen male patients with anal disease were confirmed to have HIV (97\% of total confirmed infections). HIV seroprevalence in patients with anal disease ranged from $>10 / 10,000$ in 2007 to $7.6 / 10,000$ in 2011. Among patients with nonanal disease, three were found to have HIV with an estimated seroprevalence of 0.5/10,000 in 2008 and 0.9/10,000 in 2010.

\section{HIV and/or HSV-2 seroprevalence among patients with anal disease}

As shown in Table 2, of the 2,038 male patients with anal disease, $1.8 \%$ were aged $<20$ years, $11.0 \%$ were $20-29$ years, $20 \%$ were $\geq 30$ years. Five men $(25 / 10,000)$ were diagnosed with HIV, three of whom were in their 20s, and one each in the 30-39- and 40-49-year age groups. The total HSV-2 seroprevalence was 24.0\%: $5.4 \%$ for men aged $<20$ years, $8.0 \%$ for those aged $20-29$ years, and 


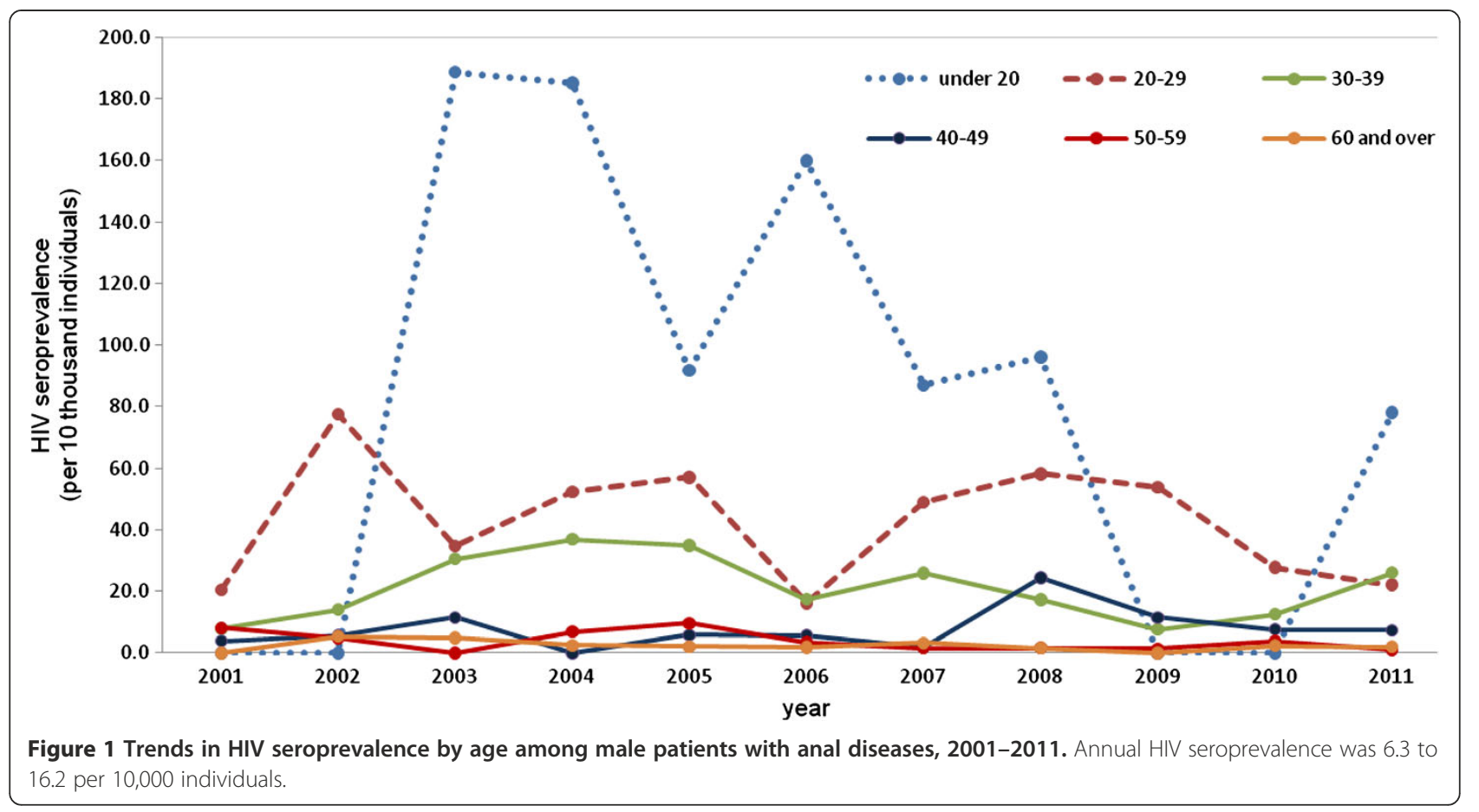

$11.7 \%$ for those aged $30-39$ years, with the rate increasing with age $(P<0.0001)$. Infection with HIV and HSV-2 was found in only one patient $(4 / 10,000)$ of the total number tested, who was in his 30s.

\section{Discussion}

In the present study, HIV seroprevalence among male patients with anal disease was between $6.3 / 10000$ and $16.2 / 10000$, which was nearly 30 times higher than that of male patients with nonanal disease at a colorectal hospital. This indicates a strong association between anal disease and HIV infection in male patients. Over the past 11 years (Figure 1), HIV seroprevalence in male patients with anal disease has decreased, although the status of HSV-2 seroprevalencehas remained at a level similar to that in the general male population.
According to the Joint United Nations Programme on HIV/AIDS (UNAIDS), HIV seroprevalence in Korea is at a low epidemic level of between $1 / 10,000$ and $4 / 10,000$ $[12,13]$. However, the number of HIV-infected individuals in Korea has continued to rise and isexpected to increase further, for two main reasons. First, the HIV epidemic pattern in Korea is similar to that observed in the early 1980s when HIV became a global pandemic. More specifically, the epidemic pattern of HIV in Korea closely follows the pattern of the early epidemiological stages of the virus when HIV was spread mainly through sexual contact, especially between men [14]. Second, in many of the recently identified cases, the infections were discovered long after onset. This means that the infected individuals may have unknowingly transmitted the virus to other people [3]. Moreover, an increasing number of HIV cases are detected during health checkups and treatment of

Table 1 Trend in HIV seroprevalence among male patients with anal disease or with nonanal disease, 2007 - 2011

\begin{tabular}{|c|c|c|c|c|c|c|}
\hline \multirow{2}{*}{ Year } & \multicolumn{3}{|c|}{ With anal diseases } & \multicolumn{3}{|c|}{ With nonanal diseases } \\
\hline & $N(\%)^{a}$ & $\mathrm{HIV}+{ }^{\mathrm{b}}$ & HIV seroprevalence per 10,000 & $\mathrm{~N}(\%)^{\mathrm{a}}$ & $\mathrm{HIV}+{ }^{\mathrm{b}}$ & HIV seroprevalence per 10,000 \\
\hline 2007 & $23,861(58.0)$ & 24 & 10.1 & $17,274(42.0)$ & 0 & 0 \\
\hline 2008 & $22,581(54.3)$ & 30 & 13.3 & $18,986(45.7)$ & 1 & 0.5 \\
\hline 2009 & $26,173(53.2)$ & 20 & 7.6 & $23,000(46.8)$ & 0 & 0 \\
\hline 2010 & $27,552(56.3)$ & 19 & 6.9 & $21,378(43.7)$ & 2 & 0.9 \\
\hline 2011 & $30,225(56.4)$ & 23 & 7.6 & $23,382(43.6)$ & 0 & 0 \\
\hline
\end{tabular}

HIV prevalence was defined as the number of confirmed HIV cases per 10,000 HIV-tested people over a 1-year period.

Nonanal diseases: patients who presented at the hospital with a nonanal disease or for a health checkup.

${ }^{\mathrm{a}} \mathrm{N}(\%)$ : the number of individuals tested for HIV (the proportion of patients with anal disease or nonanal disease).

${ }^{b} \mathrm{HIV}+$ : the number of HIV-infected individuals. 
Table 2 HIV seroprevalence and HSV-2 seroprevalence by age among male patients with anal disease, 2009

\begin{tabular}{|c|c|c|c|c|c|}
\hline Age & No. (\%) & $\mathrm{HIV}+{ }^{\mathrm{a}}$ & HIV seroprevalence per 10000 & HSV $-2+{ }^{b}$ & HSV-2 seroprevalence (\%) \\
\hline Total & $2,038(100)$ & 5 & 24.5 & 489 & 24.0 \\
\hline$<20$ & $37(1.8)$ & 0 & 0 & 2 & 5.4 \\
\hline $20-29$ & $224(11.0)$ & 3 & 134 & 18 & 8.0 \\
\hline $30-39$ & $446(21.9)$ & 1 & 22 & 52 & 11.7 \\
\hline $40-49$ & $398(19.5)$ & 1 & 25 & 85 & 21.4 \\
\hline $50-59$ & $477(23.4)$ & 0 & 0 & 166 & 34.8 \\
\hline$\geq 60$ & $456(22.4)$ & 0 & 0 & 166 & 36.4 \\
\hline
\end{tabular}

HIV seroprevalence was defined as the number of confirmed HIV cases per 10,000 of HIV-tested people.

HSV-2 seroprevalence was defined as the proportion of HSV-2-positive people. The 2,038 HSV-2 samples randomly selected among 26,173 male patients with anal disease tested for HIV in 2009.

${ }^{a} \mathrm{HIV}+$ : the number of HIV-infected individuals.

${ }^{\mathrm{b}} \mathrm{HSV} 2$ : the number of HSV-2-infected individuals.

other conditions [13]. Therefore, the role of private health-care institutions has become important in both HIV treatment and diagnosis.

In a prior study that examined HIV seroprevalence during 2002 and 2007, based on data collected from colorectal hospitals across the country, HIV seroprevalence was between 2.2/10,000 and 3.9/10,000, which was about two or three times higher than that of all hospital patients $[13,15]$. This study analyzed the characteristics of HIV seroprevalence among patients in a colorectal hospital according to variables including sex, age and presence of anal disease. The results showed that most of the cases of HIV were in patients who also had anal disease. Overall, HIV seroprevalence in patients with anal disease was $\approx 10 / 10000$. In Korea, HIV seroprevalence was highest among sexual partners of HIV-infected individuals $(>1,000 / 10,000)$, followed by people who applied voluntarily for HIV testing because they wanted to know their status $(>20 / 10,000)$, and people who underwent additional HIV tests when their doctor suspected HIV infection during treatment $(>10 / 10,000)[11,16]$. This study showed that male patients with anal disease were similar to those referred by their doctor and were assumed to be a high-risk group.

In addition, male patients in whom HIV infection was detected at the colorectal hospital tended to be younger than the general HIV-infected male population, and HIV seroprevalence increased significantly with younger age. For specific identification of high HIV seroprevalence in the group aged $<20$ years, we divided into groups aged $<10$ years, $10-14$ years and $15-19$ years. From 2001 to $2011,1,061$ male patients aged 15-19 years were treated and underwent HIV testing at the hospital. Ten HIV infections were found in the group aged 15-19 years.

In Korea, the HIV-infected young men aged 15-19 years accounted for $2.4 \%$ of the total number of HIV-infected men as of 2011. Sixty percent of these were infected through homosexual contacts, which is higher than the overall percentage infected through homosexual contact
(43\%) [17]. In the present study, male patients aged 15-19 years comprised $3.7 \%$ of the total number of male patients with HIV and anal disease. This produced a higher annual HIV seroprevalence than for any other group nationwide in Korea.

A survey of male sexual behavior in Korea showed that sexual activity began around age 16 years [18], and only $10-20 \%$ used condoms [19]. A survey of homosexual behavior in Korea showed that $60 \%$ of participants had a homosexual feeling before age 18 years [20]. It is expected that the group aged $15-19$ years is a group at high risk for STI.

Approximately $70 \%$ of HIV-infected individuals in the present study contracted the virus through homosexual contact, according to the HIV database of KCDC. In Korea, HIV infection was detected in many people who visited HIV testing and consulting centers for homosexual people (HIV-positive rate of 5.5\%) [21]. However, the proportion of homosexual men who tested for HIV over the past year in Korea was lower than that in other countries including Japan, China and the United States [22]. Moreover, nearly half of all homosexual men in Korea said that they had never tested for HIV [20]. Therefore, the present study shows that ensuring that colorectal surgeons can easily identify lesions associated with HIV infection in male patients with anal disease is important for preventing misdiagnosis or delayed diagnosis of HIV infection and stopping its spread.

HSV-2 can often cause acute infection or remain inactive until it is triggered by a decline in the immune system. HSV-2 is the cause of $>90 \%$ of anorectal infections and frequently the source of proctitis in homosexual men [23]. In homosexual men living in San Francisco, the seroprevalence of HSV-2, HIV and HSV-2/HIV was $26.1 \%, 18.6 \%$ and $12.0 \%$, respectively [24]. In male infected with HIV by sexual contacts in Korea in 2003, HSV-2 seroprevalence (23.9-69.2\%) was higher than that in the general male population (6.8-26.0\%) [9]. In the present study, $20 \%$ of HIV-infected individuals were found to have 
HSV-2. Moreover, HSV-2 seroprevalence in male patients with anal disease was similar to that in the general male population.

The present study had the following limitations. First, there is limited scope to generalize the findings of the study, because the estimates of HIV seroprevalence were based on data obtained from a single colorectal hospital located in Seoul. However, the study did show the trends, status, and characteristics of HIV seroprevalence among male patients with anal disease. Second, although the estimated HSV-2 seroprevalence in male patients with anal disease was flawed because the results were based on data from a single hospital during a limited period, the estimate is important because it is believed to be the first such study in Korea. Finally, we don't have data on sexual behavior to find out the reason of high HIV seroprevalence among male patients with anal disease. And, HSV-2 seroprevalence was low in male patients with anal disease, which differed from other studies $[25,26]$. It will be necessary to establish the relationship between the study results and the epidemiological characteristics of the study subjects in Korea.

\section{Conclusions}

The HIV seroprevalence of male patients with anal disease was significantly higher than that in patients with other diseases. The high HIV seroprevalence in patients in a colorectal hospital suggests the need to develop effective prevention programs for sexually transmitted infections, with a focus on hospitals specializing in anal diseases.

\section{Ethics statement}

Ethics approval was obtained from KCDC Institutional Review Board (IRB) Ethics Committee.

\section{Abbreviations \\ (STI): Sexual transmitted infection; (HSV-2): Herpes simplex virus type 2; (KCDC): Korea centers for diseases control and prevention; (S colorectal hospital): Songdo colorectal hospital.}

\section{Competing interest}

The authors declare that they have no competing interests.

\begin{abstract}
Authors' contributions
J-SW, DYH and SSK designed and conceived the idea for the study and M-KK supervised all aspects of its implementation. J-SW wrote the first draft of the manuscript. DYH and JKL contributed to the collection and interpretation of raw data. M-KK and $\mathrm{H}-\mathrm{KY}$ completed all data analyses and SSK coordinated funding for the project. All authors read and approved the final version of
\end{abstract} the manuscript as submitted to BMC Infectious Diseases.

\section{Acknowledgments}

This study was supported by a Grant for Health Promotion against HIV/AIDS \& STI (091-4800-4842-302) and was studied as project on HIV/AIDS seroprevalence in Korea (2006-N5100-00) of Intramural Research Grant from the Korea Centers for Disease Control and Prevention.

\section{Author details}

${ }^{1}$ Division of AIDS, Center for Immunology and Pathology, Korea Centers for Diseases Control and Prevention, Osong Health Technology Adminstration
Complex, 187 Osongsaengmyeong2(i)-ro, Cheongwon-gun,

Chungcheongbukdo 363-951, Korea. 'Division of Biobank for Health Sciences, Korea Centers for Diseases Control and Prevention, Osong,

Chungcheongbuk-do, Korea. ${ }^{3}$ Department of Surgery, Song Do Medical center, Seoul, Korea.

Received: 21 March 2013 Accepted: 3 October 2013

Published: 20 January 2014

\section{References}

1. Centers for Disease Control and Prevention: Revised guidelines for HIV counseling, testing, and referral. MMWR Recomm Rep 2001, 50(RR-19):1-57.

2. Korea Centers for Disease Control and Prevention: 2012 guideline for HIV/ AIDS Control: chapter of the surveillance reports for HIV/AIDS in Korea. KCDC 2012:15.

3. Lee JH, Kim GJ, Choi BS, Hong KJ, Heo MK, Kim SS, Kee MK: Increasing late diagnosis in HIV infection in South Korea: 2000-2007. BMC Public Health 2010, 10:411.

4. Miles AJ, Mellor CH, Gazzard B, Allen-Mersh TG, Wastell C: Surgical management of anorectal disease in HIV-positive homosexuals. Br J Sur 1990, 77:869-871.

5. Wexner SD, Smithy WB, Milsom JW, Daily TH: The surgical management of anorectal diseases in AIDS and pre-AIDS patients. Dis Colon Rectum 1986, 29:719-723.

6. Perez-Brumer AG, Konda KA, Salvatierra HJ, Segura ER, Hall ER, Montano SM, Coates TJ, Klausner JD, Caceres CF, Clark JL: Prevalence of HIV, STIs, and risk behaviors in a cross-sectional community- and clinic-based sample of men who have sex with men (MSM) in Lima, Peru. PLoS One 2013, 8(4):E59072.

7. Hwang DY, Lee JS, Yoon SG, Rhyou JH, Song SG, Lee JK: Benign anal diseases on patients with HIV disease. J Korean Soc Coloprostol 2003, 19:1-5.

8. Centers for Disease Control and Prevention: Sexually transmitted disease treatment guidelines 2002. MMWR 2002, 51(No. RR-6):11-17.

9. Shin HS, Park JJ, Chu C, Song HJ, Cho KS, Lee JS, Kim SS, Kee MK: Herpes simplex virus type 2 seroseroprevalence in Korea: rapid increase of HSV2 seroseroprevalence in the 30s in the southern part. J Korean Med Sci 2007, 22:957-962.

10. Smith JS, Robinson NJ: Age-specific prevalence of infection with herpes simplex virus types 2 and 1: a global review. J Infect Dis 2002, 186(Suppl 1):S3-28.

11. Kee MK, Lee JH, Whang J, Kim SS: Ten-year trends in HIV seroprevalence among visitors to public health centers under the National HIV Surveillance System in Korea, 2000 to 2009. BMC Public Health 2012, 12:831.

12. WHO/UNAIDS: Epidemiological fact sheet on HIV and AIDS: core data on epidemiology and response. Republic of Korea: WHO/UNAIDS; 2008. Updated December 2008

13. Lee JH, Hong KJ, Wang JS, Kim SS, Kee MK. Estimation of hospital-based HIV seroseroprevalence as a nationwide scale by novel method; 2002-2008 in Korea. BMC Public Health 2010, 10:739.

14. Shin SR: Republic of Korea. In Fighting a rising tide: the response to AIDS in East Asia. Edited by Yamamoto T, Itoh S. Tokyo: Japan Center for International Exchange; 2006:156-171. http://www.jcie.org/ researchpdfs/ RisingTide/rok.pdf.

15. Kee MK, Hwang DY, Lee JK, Kim SH, Chu C, Lee J-H, Kim SS: Estimation of HIV seroseroprevalence in colorectal hospitals by questionnaire survey in Korea, 2002-2007. Public Health Res Perspect 2011, 2(2):104-110.

16. Kee MK, Lee JH, Chu C, Lee EJ, Kim SS: Characteristics of HIV seroseroprevalence of visitors to public health centers under the national HIV surveillance system in Korea: cross sectional study. BMC Public Health 2009, 9:123.

17. Korea Centers for Disease Control and Prevention: 2011 annual Report on the notified HIV/AIDS in Korea. KCDC 2011:20-21.

18. KCDC, The report of International AIDS symposium, 2004: The social and economic impacts of HIV/AIDS infection in Korea. KCDC 2004:222-223.

19. Sohn A, Han HJ: Adolescents's sexuality and school-based sex education in South Korea. J Soc Health Educ and Prom 2000, 19(4):45-60.

20. Kee MK, Park CM, Chang CG, Go UY: Sexual Behavioral characteristics and the knowledge of HIV/AIDS among men who have sex with men in republic of Korea. J Prev Med Public Health 2004, 37(3):220-224.

21. APCASO (Asia Pacific Council of AIDS Service Organization): Men who have sex with men \& the 2011 political declaration on HIV/AIDS. Vol. 5. 2012. http:// www.apcaso.org/v2/wp-content/uploads/2011/12/InFocus-ol5-MSM.pdf. 
22. UNAIDS: AIDS info database. http://www.unaids.org/globalreport/ aidsinfo.htm

23. Gupta R, Warren T, Wald A: Genital herpes. Lancet 2007, 370(9605):2127-2137.

24. Stamm WE, Handsfield HH, Rompalo AM, Ashley RL, Roberts PL, Corey L: The association between genital ulcer disease and acquisition of HIV infection in homosexual men. JAMA 1988, 260:1429-1433.

25. Patel P, Bush T, Mayer KH, Desai S, Henry K, Overton ET, Conley L, Hammer J, Brooks JT: Prevalence and risk factors associated with herpes simplex virus-2 infection in a contemporary cohort of HIV-infected persons in the United States. Sex Transm Dis 2012, 39(2):154-160.

26. Romanowski B, Myziuk LN, Walmsley SL, Trottier S, Singh AE, Houston S, Joffe M, Chiu I: Seroprevalence and risk factors for herpes simplex virus infection in a population of HIV-infected patients in Canada. Sex Transm Dis 2009, 36:165-169.

doi:10.1186/1471-2334-14-34

Cite this article as: Wang et al: Hospital-based HIV/HSV-2

seroprevalence among male patients with anal disease in Korea: cross sectional study. BMC Infectious Diseases 2014 14:34.

\section{Submit your next manuscript to BioMed Central and take full advantage of:}

- Convenient online submission

- Thorough peer review

- No space constraints or color figure charges

- Immediate publication on acceptance

- Inclusion in PubMed, CAS, Scopus and Google Scholar

- Research which is freely available for redistribution 